\title{
Hall-magnetohydrodynamic small-scale dynamos
}

\author{
Daniel O. Gómez, ${ }^{*}$ Pablo D. Mininni, ${ }^{\dagger}$ and Pablo Dmitruk ${ }^{*}$ \\ Departamento de Física, Facultad de Ciencias Exactas y Naturales, Universidad de Buenos Aires and CONICET, \\ Ciudad Universitaria, 1428 Buenos Aires, Argentina
}

(Received 28 May 2010; published 27 September 2010)

\begin{abstract}
Magnetic field generation by dynamo action is often studied within the theoretical framework of magnetohydrodynamics (MHD). However, for sufficiently diffuse media, the Hall effect may become non-negligible. We present results from three-dimensional simulations of the Hall-MHD equations subjected to random nonhelical forcing. We study the role of the Hall effect in the dynamo efficiency for different values of the Hall parameter. For small values of the Hall parameter, the small-scale dynamo is more efficient, displaying faster growth and saturating at larger amplitudes of the magnetic field. For larger values of the Hall parameter, saturation of the magnetic field is reached at smaller amplitudes than in the MHD case. We also study energy transfer rates among spatial scales and show that the Hall effect produces a reduction of the direct energy cascade at scales larger than the Hall scale, therefore leading to smaller energy dissipation rates. Finally, we present results stemming from simulations at large magnetic Prandtl numbers, which is the relevant regime in the hot and diffuse interstellar medium. In the range of magnetic Prandtl numbers considered, the Hall effect moves the peak of the magnetic energy spectrum as well as other relevant magnetic length scales toward the Hall scale.
\end{abstract}

DOI: 10.1103/PhysRevE.82.036406

PACS number(s): 95.30.Qd, 47.27.-i, 96.50.Bh

\section{INTRODUCTION}

The generation of magnetic fields by dynamo activity plays an important role in a wide range of astrophysical objects, ranging from stars to clusters of galaxies. The gas in these objects is characterized by turbulent flows, as shown, for instance, by scintillation observations of the interstellar medium (ISM) $[1,2]$ or from pressure maps in galaxy clusters [3]. Mechanisms able to generate magnetic fields by dynamo action are often classified as large- and small-scale dynamos, depending on the correlation length of the induced magnetic field. In this context, large and small are referred to the energy-containing scale of the turbulent hydrodynamic flow. This classification is not rigid, as in many astrophysical objects both dynamos may be at work, but it gives a useful framework considering the limitations in the scale separation that can be achieved in numerical simulations. Also, the physical properties of the flows that can give rise to one or the other are somewhat different.

Helical flows have proved efficient in generating largescale dynamos, i.e., on scales larger than the energycontaining eddies of the flow [4-7]. It is now known that large-scale dynamo action can also be produced by anisotropic and inhomogeneous flows (e.g., flows with a largescale shear). On the other hand, nonhelical flows can be instrumental in generating small-scale dynamos [8], i.e., on

\footnotetext{
*Also at Instituto de Astronomía y Física del Espacio, C.C. 67 Suc. 28, 1428 Buenos Aires, Argentina; http://astro.df.uba.ar; dgomez@df.uba.ar

${ }^{\dagger}$ Also at IFIBA (Instituto de Física de Buenos Aires), CONICET, 1428 Buenos Aires, Argentina and National Center for Atmospheric Research, Boulder, CO 80307, USA.

${ }^{\ddagger}$ Also at IFIBA (Instituto de Física de Buenos Aires), CONICET, 1428 Buenos Aires, Argentina.
}

sizes smaller than those of the energy-containing eddies [9-11]. In recent years, the study of small-scale dynamos with magnetic Prandtl number $\mathrm{Pm}=\nu / \eta$ (the ratio between the viscosity and the magnetic diffusivity of the plasma) different from unity has received special attention $[12,13]$, both for $\mathrm{Pm} \gg 1[14]$ and for $\mathrm{Pm} \ll 1[15,16]$. Motivations to study these regimes include recent experiments of dynamo action using liquid sodium [17], as well as the fact that many astrophysical plasmas are characterized by magnetic Prandtl numbers different from unity. For instance, the magnetic Prandtl number is much smaller than 1 in the solar convective region, and it is typically much larger than 1 in the interplanetary medium and also in the ISM.

For sufficiently low-density media such as the one that pervades the ISM, kinetic effects such as the Hall effect or ambipolar diffusion might also become relevant [18]. The potential relevance of ambipolar diffusion in astrophysical dynamos was studied in Refs. $[19,20]$. The relevance of the Hall effect has been recognized in various astrophysical applications $[18,21,22]$, space plasmas [23-25], and also laboratory plasmas [26-28]. The role of the Hall effect on largescale dynamos subjected to helical forcing has also been addressed in the literature [29,30]. Less attention has received the impact of kinetic effects on the small-scale dynamo. A theoretical model of the kinematic small-scale dynamo with Hall effect was presented in [31]. No numerical studies of the nonlinear and saturated regime were considered in the literature.

In this paper, we present results from three-dimensional simulations of the Hall-magnetohydrodynamics (MHD) equations subjected to random nonhelical forcing. The main aim is to study the role of the Hall effect in the small-scale dynamo efficiency for different values of the Hall parameter. As a result of the study, we also discuss the impact of the Hall effect on the dynamo saturation values and on magnetic and total dissipation rates. The structure of the paper is as follows. A brief introduction to the theoretical framework 
known as Hall-MHD is presented in Sec. II. The role of the Hall effect in the efficiency of the dynamo is shown in Sec. III. In Sec. IV we characterize the stationary regime that is attained when the dynamo process saturates, showing the corresponding energy power spectra. The energy transfer rates participating in the nonlinear energy cascade are displayed in Sec. V. In Sec. VI, we explore the regime of large magnetic Prandtl number (i.e., when the viscous dissipation scale is larger than the resistive dissipation scale) which, as mentioned, is particularly relevant in diffuse media such as the ISM. Finally, the conclusions are summarized in Sec. VII.

\section{HALL-MHD EQUATIONS}

For the sake of simplicity, we consider incompressible flows, although compressible effects may be relevant, e.g., in the ISM for the formation of structures [32]. Incompressible Hall-MHD is described by the modified induction equation (i.e., with the addition of the Hall current) and the equation of motion (the Navier-Stokes equation),

$$
\begin{gathered}
\frac{\partial \boldsymbol{B}}{\partial t}=\boldsymbol{\nabla} \times[(\boldsymbol{U}-\boldsymbol{\epsilon} \boldsymbol{\nabla} \times \boldsymbol{B}) \times \boldsymbol{B}]+\eta \nabla^{2} \boldsymbol{B}, \\
\frac{\partial \boldsymbol{U}}{\partial t}=-(\boldsymbol{U} \cdot \boldsymbol{\nabla}) \boldsymbol{U}+(\boldsymbol{B} \cdot \boldsymbol{\nabla}) \boldsymbol{B}-\boldsymbol{\nabla}\left(P+\frac{B^{2}}{2}\right)+\boldsymbol{F}+\nu \nabla^{2} \boldsymbol{U},
\end{gathered}
$$

where $\boldsymbol{F}$ denotes a solenoidal and nonhelical external force, which is delta correlated in time. The velocity $\boldsymbol{U}$ and the magnetic field $\boldsymbol{B}$ are expressed in units of a characteristic speed $U_{0}=\sqrt{\left\langle U^{2}\right\rangle}, \eta$ is the magnetic diffusivity, and $\nu$ is the kinematic viscosity. The parameter $\epsilon$ measures the relative strength of the Hall effect and can be written as

$$
\epsilon=\frac{c}{\omega_{p i} L_{0}} \frac{U_{A}}{U_{0}},
$$

where $L_{0}$ is a characteristic length scale, $U_{A}=\sqrt{\left\langle B^{2}\right\rangle / 4 \pi n m_{i}}$ is the Alfven speed, and $w_{p i}=\sqrt{4 \pi e^{2} n / m_{i}}$ is the ion plasma frequency ( $e$ is the electron charge; $n=n_{e}=n_{i}$ are the particle, electron, and ion densities, respectively; and $m_{i}$ is the ion mass). Hereafter, we adopt $U_{0}=U_{A}$ as our typical velocity, thus rendering the Hall parameter simply as $\epsilon=c /\left(\omega_{p i} L_{0}\right)$, i.e., a dimensionless version of the ion skin depth. These equations are complemented by the solenoidal conditions for both vector fields, i.e.,

$$
\boldsymbol{\nabla} \cdot \boldsymbol{B}=0=\boldsymbol{\nabla} \cdot \boldsymbol{U}
$$

From a theoretical point of view, Hall-MHD corresponds to a two-fluid description of a fully ionized plasma: a positively charged ion species of mass $m_{i}$ moving with the velocity field $\boldsymbol{U}(\boldsymbol{r}, t)$ and negatively charged massless electrons with the velocity

$$
\boldsymbol{U}_{e}=\boldsymbol{U}-\boldsymbol{\epsilon} \boldsymbol{\nabla} \times \boldsymbol{B} .
$$

Therefore, from Eqs. (1)-(5) we obtain that in the ideal limit (i.e., $\eta \rightarrow 0$ ), the magnetic field is frozen to the electron flow.
As a result, advection, stretching, and folding of magnetic field lines (mechanisms relevant for dynamo action) are performed by the electron flow rather than by the bulk flow, resulting in potential modifications to magnetic field generation when the Hall effect is not negligible.

\section{LINEAR AND NONLINEAR DYNAMO EFFICIENCIES}

We performed simulations of the Hall-MHD equations with a spatial resolution of $256^{3}$ grid points, using a pseudospectral code [33]. The linear size of our numerical box is $2 \pi L_{0}$ (with $L_{0}$ as a unit length), and periodic boundary conditions in the three Cartesian directions are assumed. We apply the $2 / 3$ dealiasing rule, and therefore the maximum wave number resolved by the code is $k_{\max }=256 / 3 \approx 85$. We first consider simulations with magnetic Prandtl number equal to unity (i.e., $\mathrm{Pm}=1$ ). The coefficients of viscosity and resistivity in these simulations are set to $\nu=\eta=2 \times 10^{-3}$, which ensure that the dissipation scales are well resolved, i.e., at all times the dissipation wave numbers $k_{\nu}$ $=\left(\left\langle\omega^{2}\right\rangle / \nu^{2}\right)^{1 / 4}$ and $k_{\eta}=\left(\left\langle J^{2}\right\rangle / \eta^{2}\right)^{1 / 4}$ remain smaller than $k_{\max }$ (here, $\boldsymbol{\omega}=\boldsymbol{\nabla} \times \boldsymbol{U}$ is the vorticity and $\boldsymbol{J}=\boldsymbol{\nabla} \times \boldsymbol{B}$ is the current density). To evolve the equations in time we use a fully explicit second-order Runge-Kutta scheme. We note that for Hall-MHD, and for velocity and magnetic fields of order unity, the Courant-Friedrichs-Levy (CFL) condition becomes $\Delta t \leq(\Delta x)^{2} / \epsilon$ (due to the dispersive nature of the whistler waves), which is more restrictive than the regular CFL condition $\Delta t \leq \Delta x$. As a result, the time step decreases quadratically with the spatial resolution and also decreases linearly with the Hall parameter $\epsilon$. The Hall-MHD dynamo simulations are therefore computationally more expensive than the equivalent MHD runs, resulting in the modest spatial resolution considered here.

We first generate stationary hydrodynamic turbulence by integrating Eq. (2) subjected to random nonhelical forcing (i.e., such that $\boldsymbol{\nabla} \times \boldsymbol{F} \perp \boldsymbol{F}$ ) centered at wave numbers $|\boldsymbol{k}|$ $\approx k_{F}=3$ and delta correlated in time. Once the kinetic energy reaches a stationary regime as a result of the balance between the power delivered by the external force and viscous dissipation, the hydrodynamic simulation is stopped. In a second stage, a random and small magnetic field is introduced at small scales, and the simulation is restarted with the full Hall-MHD equations (1) and (2).

We performed simulations with different values of the Hall parameter $\epsilon$, including a purely MHD case corresponding to $\epsilon=0$. Whenever $\epsilon \neq 0$, a new spatial scale is introduced (the Hall scale), which in the spectral domain is characterized by $k_{\epsilon}=1 / \epsilon$. In this paper, we consider the cases in which $k_{\epsilon}$ falls in between the macroscopic scale $k_{F}$ (set by the external driver) and the dissipation scales $k_{\nu}$ and $k_{\eta}$, which is the relevant scenario for astrophysical plasmas such as the interstellar media. In such media, the Hall scale is several orders of magnitude smaller than the largest scales, and the Hall effect can be expected to be relevant only at the smallest dynamical scales. Note, however, that these arguments ought to be regarded as motivations. Although the ordering of typical length scales is the correct one, a realistic separation of scales is completely out of reach with present computing power. 


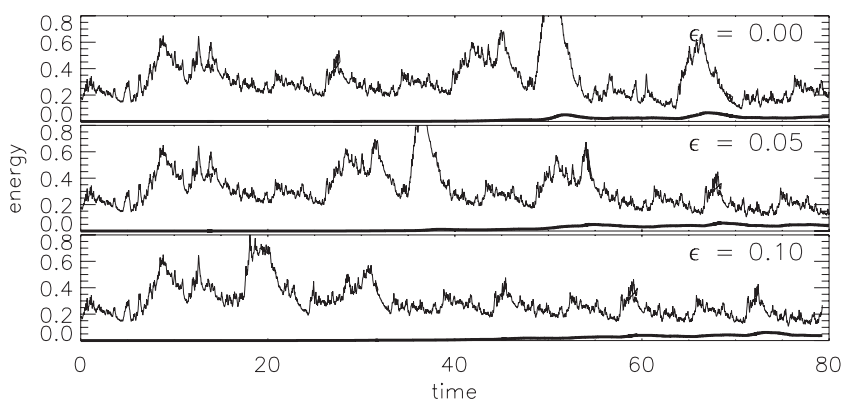

FIG. 1. Kinetic (thin lines) and magnetic (thick lines) energies vs time for $\epsilon=0,0.05$, and 0.10 (from top to bottom).

In Fig. 1 we show the statistically stationary time series for kinetic energy (thin lines) for runs with different values of the Hall parameter $\epsilon$. The magnetic energy in these runs (thick line) is observed to rise until it saturates at values which remain a moderate fraction of the corresponding kinetic energy. The viscous (thin line) and resistive (thick) dissipation rates vs time are shown in Fig. 2 for three runs with different values of the Hall parameter. In all these plots, the magnetic dissipation rate is observed to grow until it becomes fully comparable to the corresponding viscous dissipation rate (even when the kinetic energy is larger than the magnetic energy).

The exponentially fast growth of magnetic energy is shown using a linear-logarithmic scale in Fig. 3 for the same three runs. Note that there is an initial stage where the magnetic field starts growing exponentially fast, regardless of the particular value of the Hall parameter $\epsilon$. During this early stage of the dynamo, the electron flow is still approximately equal to the ion flow, i.e., $\boldsymbol{U}_{e} \approx \boldsymbol{U}$ [see Eq. (5)]. Keeping in mind that the growing magnetic field remains approximately frozen (note that this is strictly valid only in the limit $\eta$ $\rightarrow 0$ ) to the electron velocity field, we can anticipate that at some point in time the electron and ion flows will start drifting apart from one another. Therefore, a second stage arises corresponding to a nonlinear dynamo (although still "kinematic," in the sense that the magnetic field does not affect the bulk velocity field) since the magnetic field is being advected by the electron flow which at that point becomes a function of the magnetic field itself. In Fig. 3 we see that although the case $\epsilon=0$ can be approximated by a linear growth rate (indicated by the dotted straight line) all the way up to the saturation level, we cannot do the same for the cases $\epsilon=0.05$ and

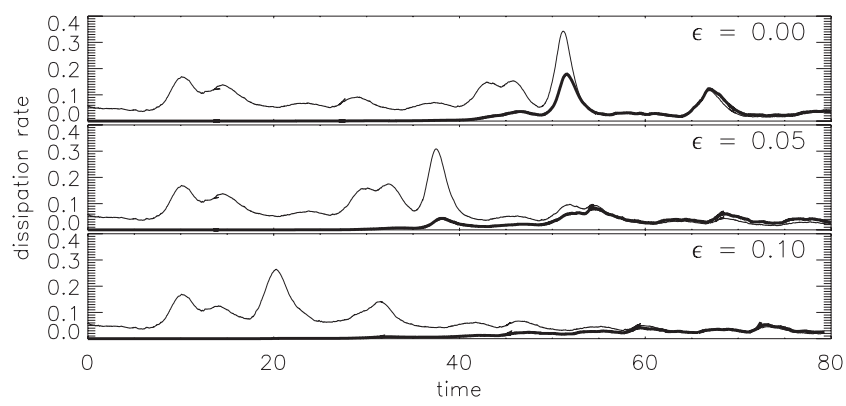

FIG. 2. Kinetic (thin lines) and magnetic (thick lines) dissipation rates vs time for $\epsilon=0,0.05$, and 0.10 (from top to bottom).

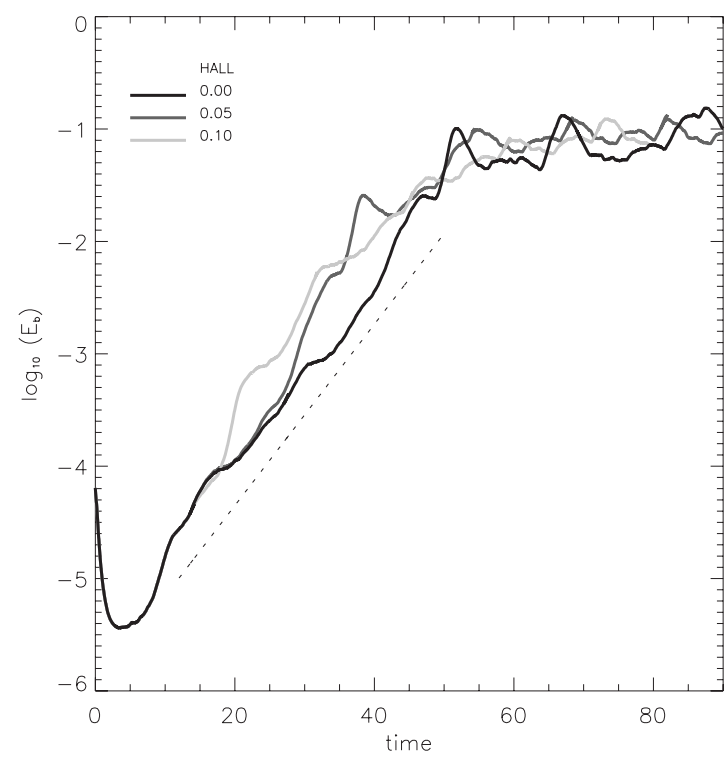

FIG. 3. Magnetic energy vs time, showing the exponential growth rate during the early linear dynamo regime.

0.10 since there is a break in the corresponding growth rates. This break occurs first for the case with larger Hall effect (i.e., $\epsilon=0.10$ ), but the incremented slope is larger for the case $\epsilon=0.05$. The fact that the dynamo efficiency improves up to a certain value of the Hall parameter and then starts decreasing is reminiscent of similar results reported in Ref. [33] for large-scale Hall-MHD dynamos.

To show the relative importance of the Hall term in the electron velocity field, in Fig. 4 we display the ratio between $\epsilon\left|J_{k}\right|$ and $\left|U_{k}\right|$ at different labeled times, where $\left|J_{k}\right|$ and $\left|U_{k}\right|$ are, respectively, the spectral intensities of the current density and of the velocity field at wave number $k$ [note that $\boldsymbol{U}_{e}=\boldsymbol{U}-\boldsymbol{\epsilon} \boldsymbol{J}$ from Eq. (5)]. The upper frame corresponds to the run with $\epsilon=0.05$ and the lower frame to $\epsilon=0.10$. The vertical gray line in each frame corresponds to the Hall scale $k_{\epsilon}$ $=1 / \epsilon$. In both cases, the Hall term becomes gradually nonnegligible and eventually dominant at the largest wave numbers of the system, i.e., $\epsilon\left|J_{k}\right|>\left|U_{k}\right|$ at $k>k_{\epsilon}$. For the case $\epsilon$ $=0.05$ (upper panel), the Hall term $\epsilon\left|J_{k}\right|$ becomes comparable to $\left|U_{k}\right|$ at the largest wave numbers by about $t \approx 24$, while a similar situation arises for $\epsilon=0.10$ at $t \approx 18$ (lower panel). These values of time are remarkably consistent with those observed in Fig. 3 for the departure from the linear regime in each of the runs.

During the linear dynamo regime, i.e., before the curves cross the line $\epsilon\left|J_{k}\right| /\left|U_{k}\right|=1$, they can be approximated by a $\sim k^{11 / 6}$ power law. This result can easily be obtained, assuming a relatively flat magnetic power spectrum [i.e., $E_{b}(k)$ $\left.\simeq k^{0}\right]$ and a Kolmogorov power spectrum for the kinetic energy $\left[E_{u}(k) \simeq k^{-5 / 3}\right]$. Furthermore, these curves collapse into one if normalized by the square root of the magnetic energy exponential growth $e^{\sigma t}$ (where $\sigma$ is the growth rate in the MHD case). After the curves cross $\epsilon\left|J_{k}\right| /\left|U_{k}\right|=1$, these two features are gradually lost since the Hall term becomes nonnegligible. This further confirms the departure from MHD behavior when the Hall current becomes on the order of the bulk velocity or, in other words, when the electron velocity 

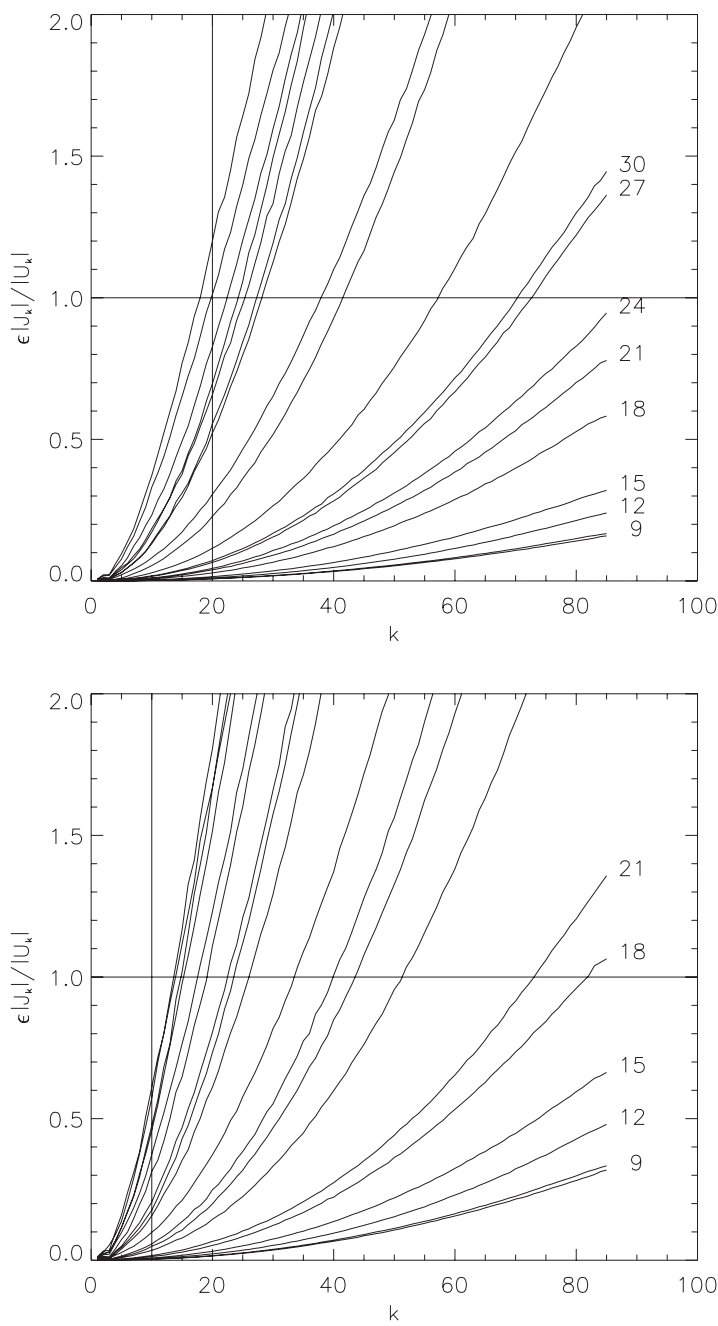

FIG. 4. Ratio between $\epsilon\left|J_{k}\right|$ and $\left|U_{k}\right|$ at different times (labeled). The top frame corresponds to the run with $\epsilon=0.05$ and the bottom frame to $\epsilon=0.10$. The vertical gray line in each frame corresponds to the Hall wave number $k_{\epsilon}=1 / \epsilon$.

starts to differ from the bulk velocity. Also, at least during the early stages of the dynamo (for which $\epsilon\left|J_{k}\right|<\left|U_{k}\right|$ ), the ratio $\epsilon\left|J_{k}\right| /\left|U_{k}\right|$ is simply proportional to $\epsilon$, reflecting the fact that the dynamics of the fields is still approximately independent of the Hall parameter.

In all these cases, there is a third and last stage, which corresponds to the saturation of the dynamo. We discuss the energy saturation levels in the next section.

\section{ENERGY SPECTRA}

The distribution of kinetic and magnetic energies among spatial scales can be observed in the energy power spectra $E_{k}$ vs $k$ displayed in Fig. 5 for three different values of the Hall parameter $\left(E_{k}\right.$ is defined such that the total energy is $E$ $=\int d k E_{k}$, and magnetic and kinetic energies are such that $\left.E_{b}+E_{u}=E\right)$. The thick line in all these runs corresponds to the total-energy spectrum, which remains in a roughly statistically stationary regime. Note that for these three runs, the kinetic-energy spectrum remains close to Kolmogorov, i.e.,
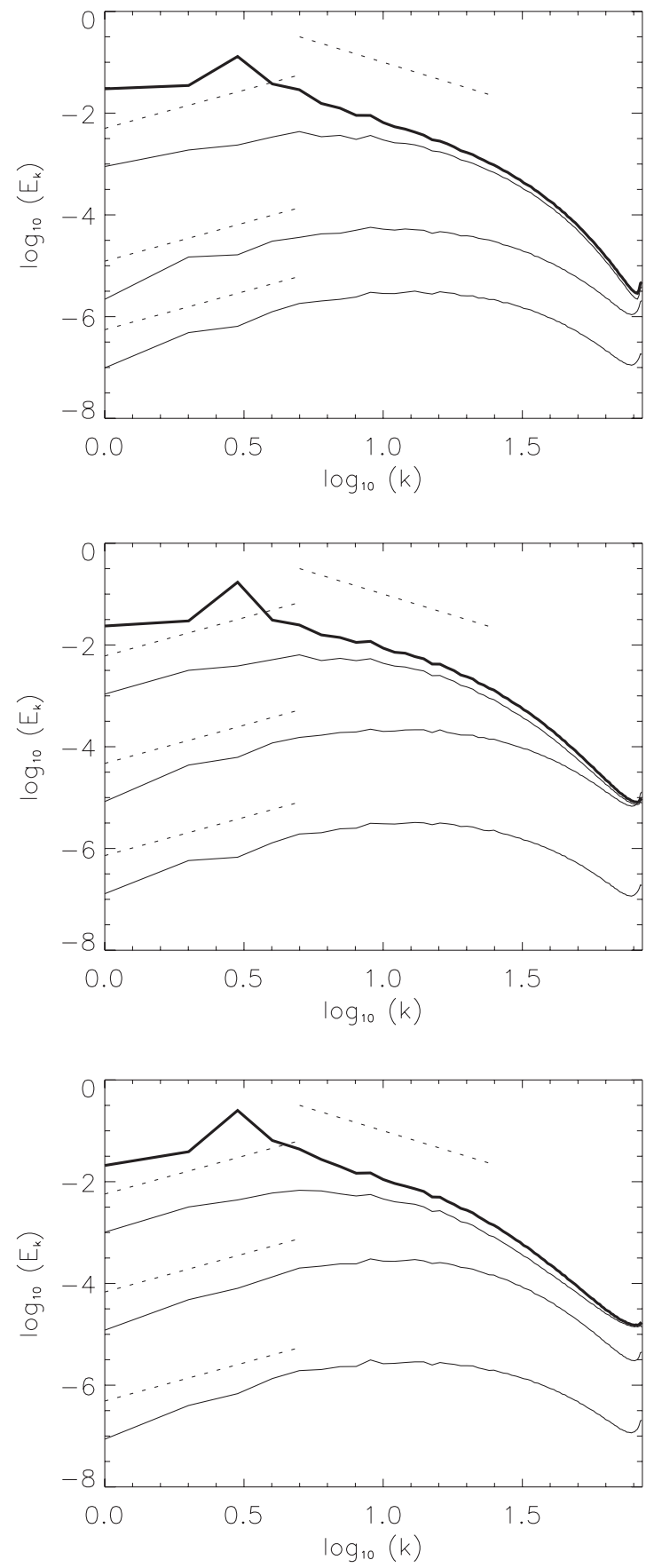

FIG. 5. Total-energy spectrum (thick trace) at $t=72$ for $\epsilon=0.00$ (top), $\epsilon=0.05$ (center), and $\epsilon=0.10$ (bottom). In each frame magnetic energy spectra at $t=18,36,72$ are also shown (corresponding to the thin lines from bottom to top). The Kolmogorov and Kazantsev spectra are overlaid (dotted traces) for reference.

$E_{k} \propto k^{-5 / 3}$, in the energy inertial range. The thin lines display magnetic energy spectra at different times, showing the growth of magnetic energy as a whole at early times, and saturation of magnetic field growth at small scales at intermediate and late times. Note that the peak of the magnetic energy in all these cases remains at wave numbers larger than the one where the external force operates (i.e., $k_{F}=3$ ), as expected for a small-scale dynamo. 
TABLE I. Global results for runs with different values of the Hall parameter $\epsilon$. $E$ is the mean saturation level of the total energy, $E_{b} / E$ is the ratio of magnetic to total energy, $k_{J}$ is the average wave number for the current density distribution, $D$ is the total dissipation rate, $D / D_{0}$ is the normalized dissipation rate, and $D_{b} / D$ is the ratio of magnetic to total dissipation rate.

\begin{tabular}{ccccccc}
\hline \hline$\epsilon$ & $E$ & $E_{b} / E$ & $k_{J}$ & $D$ & $D / D_{0}$ & $D_{b} / D$ \\
\hline 0.00 & 0.37 & 0.14 & 23.1 & 0.13 & 0.88 & 0.48 \\
0.05 & 0.35 & 0.13 & 19.4 & 0.11 & 0.78 & 0.39 \\
0.10 & 0.33 & 0.13 & 17.4 & 0.10 & 0.73 & 0.34 \\
\hline \hline
\end{tabular}

The Kazantsev slope $E_{k} \propto k^{3 / 2}[8]$ provides a reasonable approximation at small wave numbers for all these cases, even though Kazantsev's spectrum was originally derived for the kinematic regime. Kazantsev's dynamo theory [8] assumes a random velocity field with Gaussian statistics, which is spatially homogeneous and isotropic and $\delta$ correlated in time. Under these assumptions, the two-point magnetic field correlation function can be analytically computed (see Ref. [8], and also Ref. [34]), and a $k^{3 / 2}$ power law is asymptotically expected for the magnetic energy spectrum at the lowwave-number end. Even though Kazantsev's model was devised for pure MHD (no Hall effect) and for large magnetic Prandtl numbers, our Hall-MHD simulations also reproduce an $E_{k} \propto k^{3 / 2}$ magnetic spectrum equally well. This is to be expected since the Hall effect becomes negligible at the lowest wave numbers (i.e., at $k \ll k_{\epsilon}=1 / \epsilon$ ). Kazantsev's spectrum has also been reported in simulations of small-scale MHD dynamos at unity Prandtl numbers [11]. The extension of Kazantsev's model to Hall-MHD in Ref. [31] also recovers this spectrum in the regime considered here.

In summary, a preliminary inspection of the magnetic energy power spectra at early times shows no noticeable differences between MHD and Hall-MHD. On the one hand this is not surprising since the Hall effect is nonlinear in the magnetic field, and the magnetic energy at early times is much smaller than the kinetic energy at all scales. On the other hand, in what follows we show that this last conclusion is somewhat premature since there are other aspects of these turbulent dynamos that clearly show the consequences of the Hall effect.

At saturation, the total magnetic energy reaches a sizable fraction of the total kinetic energy, which can be estimated within $10-20 \%$. More specifically, after taking time averages between $t=60$ and $t=80$ (see Figs. 2 and 3), we obtain the energy ratios $E_{b} / E$ listed in Table I. Note that the saturation level of these small-scale dynamos, defined as the fraction of magnetic energy to total energy in the stationary regime, decreases with the Hall parameter. Therefore, although in the nonlinear dynamo regime the growth increases with the Hall parameter $\epsilon$, the magnetic field reaches a smaller saturation level.

As mentioned in Sec. III, the dynamics of the largest wave numbers in our simulations is controlled by viscosity and electric resistivity. Therefore, the dissipation of magnetic energy mostly takes place in current sheets with a thickness which can be expected to be close to the inverse of $k_{\eta} \approx 85$.

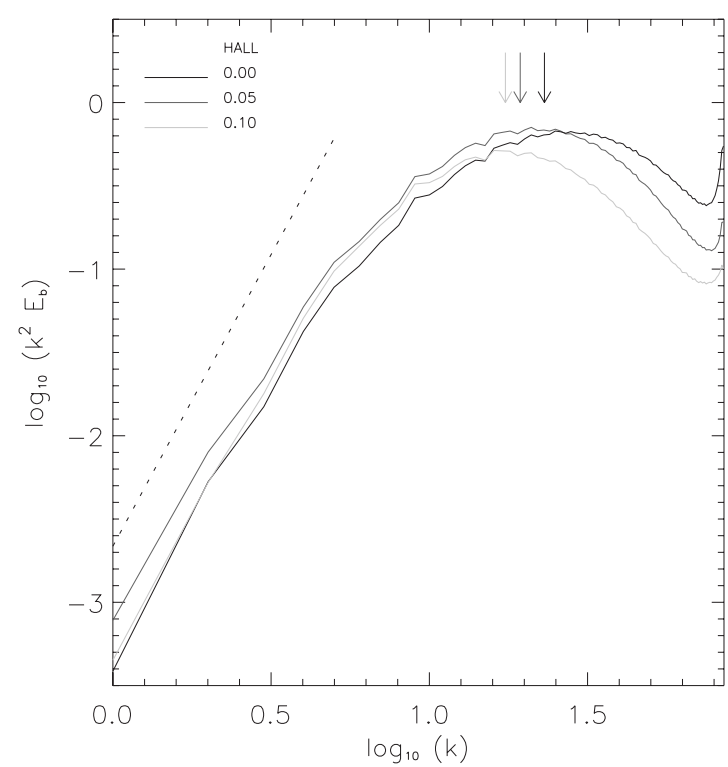

FIG. 6. Spectral distribution of current density, i.e., $k^{2} E_{b}(k)$ vs $k$ for three different values of the Hall parameter (labeled). The dotted trace corresponds to the Kazantsev slope $k^{7 / 2}$. The arrows indicate the average wave number $k_{J}$ [see Eq. (6)] for each distribution.

On the other hand, the width and the length of these current sheets will vary from one to another $[13,35]$. We can obtain a statistical average of the dimensions of our magnetic dissipative structures by computing the power spectrum of the electric current density, which is simply $k^{2} E_{b}(k)$. In Fig. 6 we show time averaged (between $t=60$ and $t=80$ ) current density spectra for three different values of the Hall parameter (labeled). All of these spectra are compatible with a Kazantsev law at low wave numbers. Note that the maximum of these spectra shifts toward smaller wave numbers as the Hall parameter increases. Since the peak of the spectrum can be associated with an average thickness of the current sheets, the above-mentioned shift can be interpreted as the current sheets becoming relatively "thicker" as the Hall effect increases. This result is in agreement with previous experimental and numerical results suggesting that in Hall-MHD the thickness of the current sheets is given by the Hall scale rather than by the Ohmic dissipative scale as in the MHD case (see [36] for recent results in support of this interpretation).

For each of these runs, we also compute the magnetic Taylor wave number $k_{J}$ given by

$$
k_{J}^{2}=\frac{\int d k k^{2} E_{b}(k)}{\int d k E_{b}(k)},
$$

which are indicated in Fig. 6 by arrows and are observed to remain close, but somewhat to the left of the maximum for the corresponding power spectrum. The magnetic Taylor scale (i.e., the inverse of $k_{J}$ ) can be interpreted as the mean curvatures of the magnetic field lines [13] and of the ensuing current sheets. The value of $k_{J}$ also moves toward smaller 
wave numbers as the Hall scale is increased. The values of $k_{J}$ for each of these runs are listed in Table I. In Table I we also list the time-averaged total dissipation rate $D=D_{u}+D_{b}$, clearly showing a progressive reduction as the Hall parameter is increased. We also list the energy dissipation rate normalized by $D_{0}=U_{0}^{3} / L_{0}$, where $U_{0}=\sqrt{\left(2 E_{u} / 3\right)}$ is the onedimensional rms velocity, and $L_{0}$ is the integral scale of the flow. This normalization is often used in the study of hydrodynamic turbulence, and the dissipation rate is then known to asymptotically reach a value close to $\approx 0.5$ [37] for Taylorbased Reynolds numbers $R_{\lambda}>200$. The normalized dissipation rates here are larger than in the hydrodynamic case, and this can be ascribed to two different causes. On one hand, we have a lower resolution than the simulations in [37], and moreover the asymptotic value of the dissipation rate in MHD turbulence seems to be obtained for even larger values of $R_{\lambda}$ [38]. On the other hand, in MHD there is Ohmic dissipation besides viscous dissipation, thus in principle allowing for larger energy dissipation rates than in hydrodynamics. So the normalization for the dissipation rate may require extra contributions than just the estimated flux due to the convective term. The ratio of magnetic to total dissipation $D_{b} / D$ also reduces as $\epsilon$ increases, going from approximate equipartition in the MHD case to about $33 \%$ for $\epsilon=0.10$, even though in all these simulations the relative content of magnetic energy $E_{b} / E$ is comparatively much smaller.

\section{ENERGY TRANSFER RATES}

Interpretation of these results on the energy dissipation rate requires a detailed analysis of the transfer and conversion rate of energy among scales and between the velocity and magnetic fields, in order to identify the sources of smallscale dynamo action in MHD and in Hall-MHD turbulence. Equations (1) and (2) are known [39] to have three ideal invariants: energy, magnetic helicity, and hybrid helicity. These are transferred between scales without losses by the nonlinear terms in Eqs. (1) and (2). In this paper we focus our attention on the transfer and conversion of energy,

$$
E=\frac{1}{2} \int d^{3} r\left(|\boldsymbol{U}|^{2}+|\boldsymbol{B}|^{2}\right)=\int d k E_{k},
$$

since the nonhelical dynamo does not generate helical magnetic fields. The dynamo process in this case is basically the conversion of mechanical energy into magnetic energy by induction to sustain the magnetic fields against Ohmic dissipation. The nonlinear terms in Eqs. (1) and (2) only redistribute energy (and the other ideal invariants) among different spatial scales, in such a way to guarantee the global invariance except for dissipative losses. To study the energy transfer in Fourier space, we follow the method of shell filters originally developed for MHD [40,41], which has more recently been extended for Hall-MHD [42]. For a given vector field $\boldsymbol{f}(\boldsymbol{r})$, we define $\boldsymbol{f}_{\kappa}(\boldsymbol{r})$ to be the composition of all wave numbers between $\kappa$ and $\kappa+1$, i.e.,

$$
\boldsymbol{f}_{\kappa}(\boldsymbol{r})=\sum_{|\boldsymbol{k}|=\kappa}^{\kappa+1} \hat{\boldsymbol{f}}(\boldsymbol{k}) e^{i \boldsymbol{k} \cdot \boldsymbol{r}},
$$

so that $\boldsymbol{f}(\boldsymbol{r})=\sum_{\kappa=0}^{\infty} \boldsymbol{f}_{\kappa}(\boldsymbol{r})$. From Eqs. (1) and (2) we can derive detailed balance equations for the kinetic and magnetic energies in the $\kappa$ shell:

$$
\begin{aligned}
& \partial_{t} E_{U}(\kappa)=\int d^{3} r\left\{\sum_{Q}[\overbrace{-\boldsymbol{U}_{\kappa} \cdot(\boldsymbol{U} \cdot \boldsymbol{\nabla}) \boldsymbol{U}_{Q}}^{\text {Advection }}+\overbrace{\boldsymbol{U}_{\kappa} \cdot(\boldsymbol{B} \cdot \boldsymbol{\nabla}) \boldsymbol{B}_{Q}}^{\text {Lorentz }}]\right. \\
& +\overbrace{\nu \boldsymbol{U} \cdot \nabla^{2} \boldsymbol{U}_{\kappa}}^{\text {Dissipation }}+\overbrace{\boldsymbol{F} \cdot \boldsymbol{U}_{\kappa}}^{\text {Injection }}\} \\
& \partial_{t} E_{B}(\kappa)=\int d^{3} r\left\{\sum_{Q}[\underbrace{-\boldsymbol{B}_{\kappa} \cdot(\boldsymbol{U} \cdot \boldsymbol{\nabla}) \boldsymbol{B}_{Q}}_{\text {Advection }}+\underbrace{\boldsymbol{B}_{\kappa} \cdot(\boldsymbol{B} \cdot \boldsymbol{\nabla}) \boldsymbol{U}_{Q}}_{\text {Induction }}]\right. \\
& +\underbrace{\eta \boldsymbol{B} \cdot \nabla^{2} \boldsymbol{B}_{\kappa}}_{\text {Dissipation }}+\underbrace{\epsilon \boldsymbol{J}_{\kappa} \cdot\left(\boldsymbol{B} \times \boldsymbol{J}_{Q}\right)}_{\text {Hall }}\} \text {. }
\end{aligned}
$$

All cubic terms in Eqs. (9) and (10) (before summing over $Q)$ can be interpreted as energy transfer from the third field in the $Q$ shell to the first field in its $\kappa$ shell (except for the Hall term, which corresponds to transfer of magnetic energy in the $Q$ shell to magnetic energy in the $\kappa$ shell), and associated with different physical processes as indicated in the equations. Note that if the sum is not performed, each term depends on two wave numbers, $\kappa$ and $Q$. The two advection terms preserve the energy by themselves, in the sense that all energy received by the field on the left (in the shell $\kappa$ ) is provided by the field on the right (in the shell $Q$ ). This is also true for the Hall term. The Lorentz and induction terms do not preserve energy by themselves, and only preserve it when considered together, as work done against the Lorentz force in Eq. (9) corresponds to magnetic induction in Eq. (10). This is expressed by the following relation [41]:

$$
\int d^{3} r \boldsymbol{B}_{\kappa} \cdot(\boldsymbol{B} \cdot \boldsymbol{\nabla}) U_{Q}=-\int d^{3} r \boldsymbol{U}_{Q} \cdot(\boldsymbol{B} \cdot \boldsymbol{\nabla}) \boldsymbol{B}_{\kappa},
$$

which states that the amount of energy received by the magnetic field in the $\kappa$ shell from the velocity field in the $Q$ shell is equal to the amount of energy removed from the velocity field in the $Q$ shell by the magnetic field in the $\kappa$ shell. As a result, the Lorentz and induction shell-to-shell transfer functions are in practice the same. If these functions are summed over all wave numbers $Q$, the transfer functions discussed in [6] (which depend only on $\kappa$ ) are obtained.

Figure 7(a) shows the total shell-to-shell energy transfer rate [i.e., the sum of all cubic terms in Eqs. (9) and (10)] in the steady state of the run with $\epsilon=0.05$. Light-gray (darkgray) contours correspond to positive- (negative-) energy transfer regions on the $(\kappa, Q)$ plane, located at fractions of $[0.001,0.010,0.100]$ of the maximum positive (minimum 


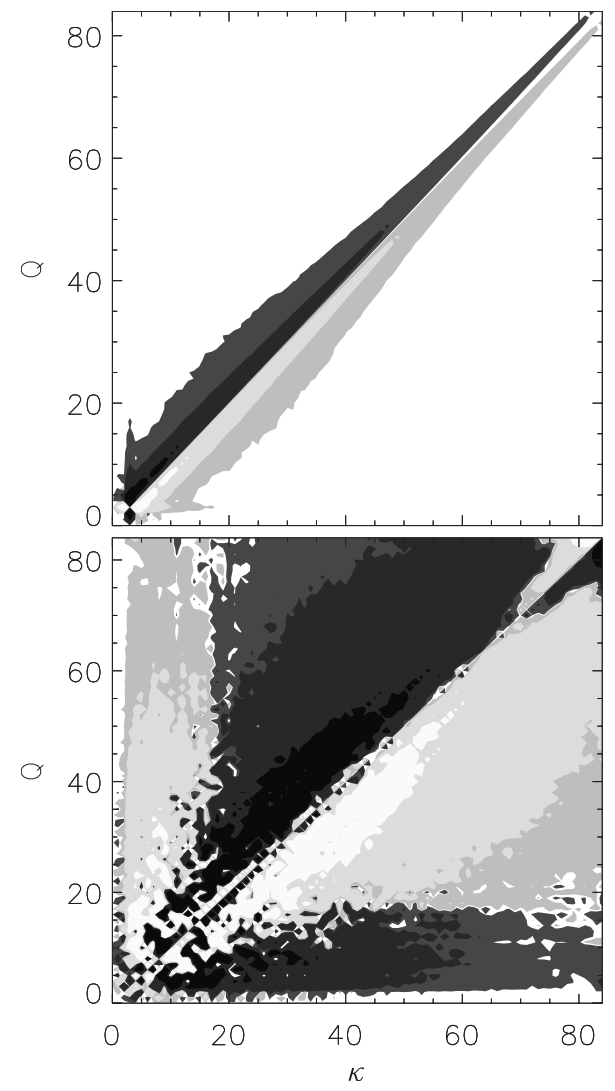

FIG. 7. Energy transfer rate contour plots on the $(\kappa, Q)$ plane for the run with $\epsilon=0.05$ at the stationary regime. Both $\kappa$ and $Q$ run from zero to $k_{\max }=85$. Light-gray filled contours correspond to levels at $[0.001,0.010,0.100]$ of the maximum positive value, while dark-gray contours display the same levels at negative energy transfer rates. The top frame shows the total transfer rate, with a peak value of 0.36 , and the bottom frame shows the Hall transfer rate, with a peak value of $4 \times 10^{-4}$

negative) value. The first thing to notice is that the integral of this function on the $(\kappa, Q)$ plane amounts to zero, which is expected to be the case for any conserved quantity in the ideal limit. The second aspect to notice is the high degree of concentration around the region $Q \approx \kappa$, which is indicative of the mostly local nature of the direct cascade of total energy in Hall-MHD, just as for MHD turbulence (for a detailed study of local and nonlocal contributions to the cascade in MHD and Hall-MHD, see Refs. [41,42]). In Fig. 7(b) we show the same kind of plot for just the Hall cubic term [see Eq. (10)]. The Hall transfer is nonlocal, although it is important to emphasize that this term is much smaller than the total transfer rate. The maximum value for the Hall transfer rate is only $4 \times 10^{-4}$, while the total transfer rate peaks at about 0.36 .

The light-gray spot on the left part of Fig. 7(b), for $\kappa, Q$ $<k_{\epsilon}=20$, indicates that energy is transferred backward by the Hall term from small scales to scales larger than the Hall scale (light-gray means positive, indicating a gain in magnetic energy). On the other hand, the light spot below the diagonal for $\kappa, Q>k_{\epsilon}=20$ indicates that at scales smaller than the Hall scale, the Hall term contributes to the direct energy cascade increasing the transfer rate toward smaller scales.
We can also compute the energy flux at the wave number $|\boldsymbol{k}|=k$ by simply performing

$$
\begin{aligned}
\Pi(k)= & \sum_{\kappa=0}^{k} \sum_{Q} \int d^{3} r\left[-\boldsymbol{U}_{\kappa} \cdot(\boldsymbol{U} \cdot \boldsymbol{\nabla}) \boldsymbol{U}_{Q}+\boldsymbol{U}_{\kappa} \cdot(\boldsymbol{B} \cdot \boldsymbol{\nabla}) \boldsymbol{B}_{Q}\right. \\
& \left.-\boldsymbol{B}_{\kappa} \cdot(\boldsymbol{U} \cdot \boldsymbol{\nabla}) \boldsymbol{B}_{Q}+\boldsymbol{B}_{\kappa} \cdot(\boldsymbol{B} \cdot \boldsymbol{\nabla}) \boldsymbol{U}_{Q}+\boldsymbol{\epsilon} \boldsymbol{J}_{\kappa} \cdot\left(\boldsymbol{B} \times \boldsymbol{J}_{Q}\right)\right] .
\end{aligned}
$$

Each of the five terms on the right-hand side of Eq. (12) has a straightforward interpretation. The first term (hereafter called $\left.\Pi_{U U}\right)$ is a purely kinetic-energy flux, which is already present in hydrodynamic turbulence, and is responsible of the direct energy cascade in that particular case. The second and fourth terms add to zero (i.e., $\Pi_{U B}+\Pi_{B U}=0$ ) since they correspond to the exchange between kinetic and magnetic energies by Lorentz forces and Faraday induction. The third term $\left(\Pi_{B B}\right)$ is flux of magnetic energy associated with the advection of magnetic field lines by the velocity field, already present in the MHD case. Finally, the fifth term $\left(\Pi_{B B}^{\text {Hall }}\right)$ is also a flux of magnetic energy, but exclusively due to the nonlinearity introduced by the Hall current. The first frame of Fig. 8 shows the total-energy flux vs $k$ for the runs with $\epsilon=0$ (black line) and $\epsilon=0.05$ (gray line). The next three frames disaggregate the energy flux into the various parts listed above.

The energy fluxes displayed in Fig. 8 confirm the observation made when considering the shell-to-shell transfer functions. Note that these energy fluxes have been normalized by the (time-averaged) total dissipation rate to allow a comparison between runs with different Hall parameter values. The flux associated with the Hall term slows down the cascade of magnetic energy toward small scales for wave numbers smaller than the Hall wave number since $\Pi_{B B}^{\text {Hall }}$ is negative in this range. At the same time, the Hall effect speeds up the energy cascade at smaller scales, where $\Pi_{B B}^{\text {Hall }}$ becomes positive. Note that the change of sign takes place at the Hall scale (see the dashed line in the lowest panel of Fig. 8). This result explains why the current spectrum was observed to peak near the Hall scale and the associated decrease in the magnetic energy dissipation rate as the amplitude of the Hall term was increased. The slowdown in the transfer of magnetic energy toward small scales (where it finally dissipates) is also responsible for the faster build up of magnetic energy through dynamo action in the Hall-MHD case.

Note that although the Hall transfer rate is three orders of magnitude smaller than the total-energy transfer rate (see Fig. 7), the contribution of the Hall term to the flux is not negligible. This is the result of the strong nonlocality of the Hall term: while the Hall transfer is small for each value of $\kappa$ and $Q$, the flux at the wave number $k$ results from summing over all values of $Q$ and over all values of $\kappa<k$. The slow decay of the Hall transfer far from the diagonal $\kappa=Q$ in Fig. 7 (associated with the nonlocality) gives a substantial contribution to the total flux.

The other fluxes are also modified by the Hall term. The total (i.e., $\Pi$ ) and $\Pi_{U U}$ fluxes decay faster with increasing wave number in the Hall-MHD case and show the buildup of 

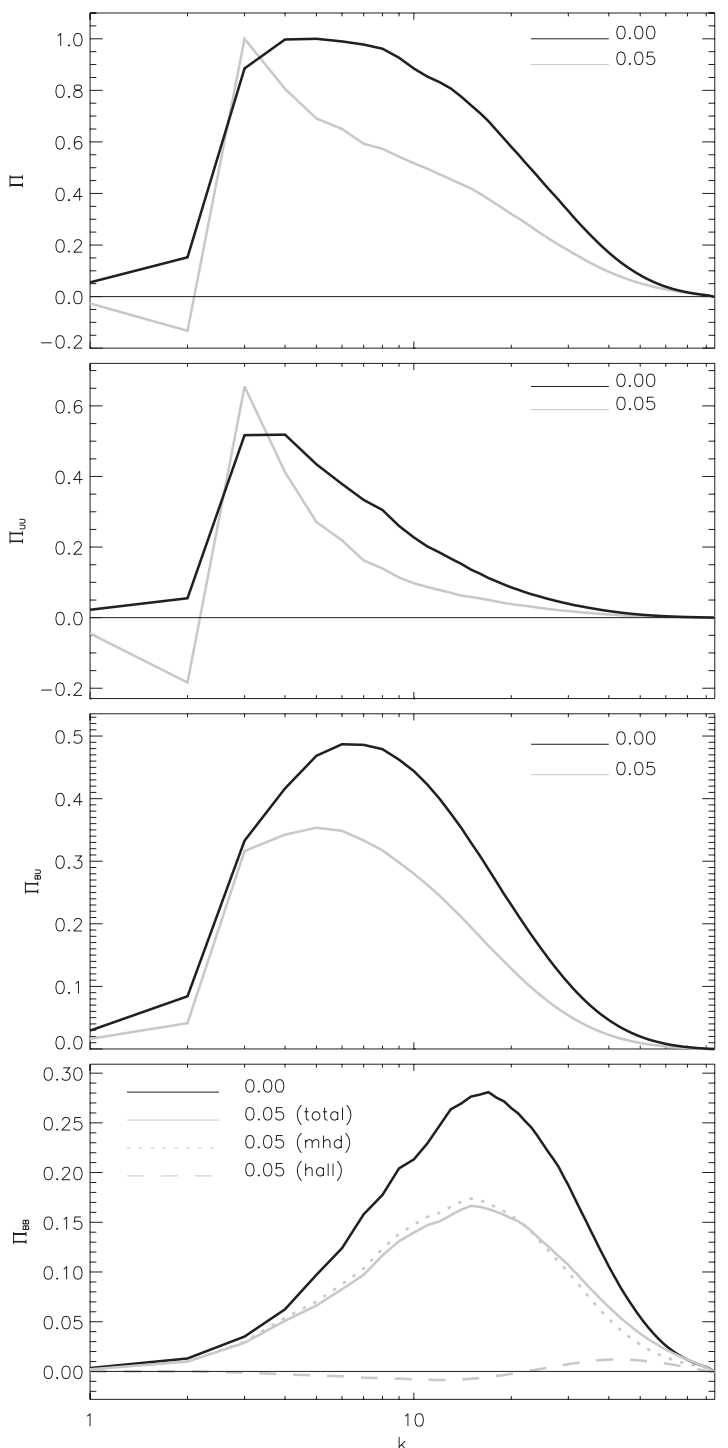

FIG. 8. Energy fluxes vs $k$, normalized by the total dissipation rate $D$. The black line corresponds to a time average at the stationary regime of the run with $\epsilon=0$, while the gray line is for $\epsilon=0.05$.

(mechanical) flows at scales larger than the forcing scale (indicated by the negative value of $\Pi_{U U}$ for small wave numbers). Such an effect for Hall-MHD has been predicted from theoretical models [43] and confirmed by numerical simulations [33]. Also, the $\Pi_{U B}$ flux peaks at smaller wave numbers in the Hall-MHD case.

The modification of the fluxes is consistent with the changes in the global dissipation rates. As the Hall term reduces the transfer of magnetic energy to smaller scales at scales larger than the Hall scale, the global dissipation of magnetic energy decreases. Note that this result is compatible with studies [44,45], which find faster reconnection rates in Hall-MHD simulations with current sheets initially set up at small scales. For spatial structures such as current sheets, at scales smaller than the Hall scale, the Hall term increases the transfer toward even smaller scales. As a result, the dissipation rate at those particular scales is also increased, which is opposite to the result obtained for the global dissipation rate.
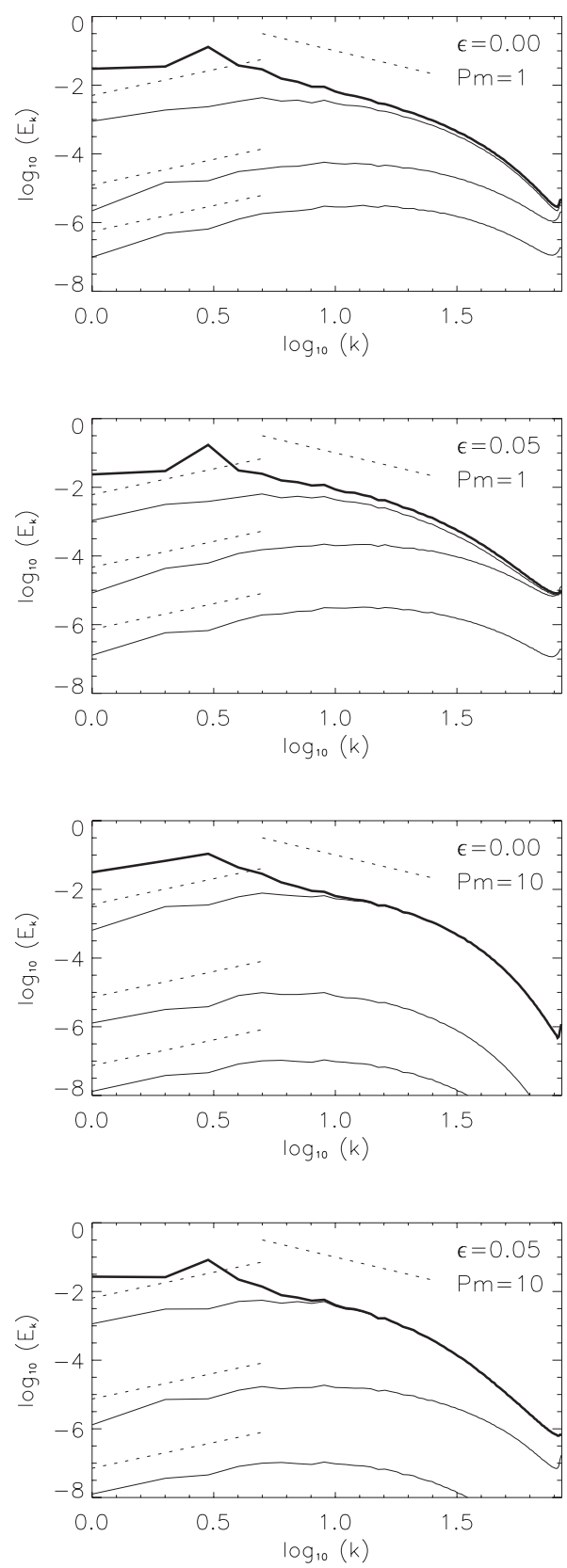

FIG. 9. Total-energy spectrum (thick trace) at $t=72$ for different runs (as labeled). Magnetic energy spectra at $t=18,36,72$ (thin lines from bottom to top in each panel) are also shown. The Kolmogorov and Kazantsev slopes are overlaid (dotted trace) for reference.

\section{LARGE MAGNETIC PRANDTL NUMBER}

We have so far considered the particular case $\mathrm{Pm}=1$, i.e., $\eta=\nu$. However, there are several low-density and hightemperature astrophysical plasmas which are characterized by $\mathrm{Pm}=\nu / \eta \gg 1$, such as the interstellar medium, intracluster gas in between of galactic clusters, jets, or accretion disks. We performed numerical simulations with $\mathrm{Pm}=10$, so that the viscous dissipation wave number $k_{\nu}$ becomes much smaller than the resistive dissipation wave number $k_{\eta}$. As a result, magnetic fluctuations in this large-Pm regime may grow at subviscous scales. 


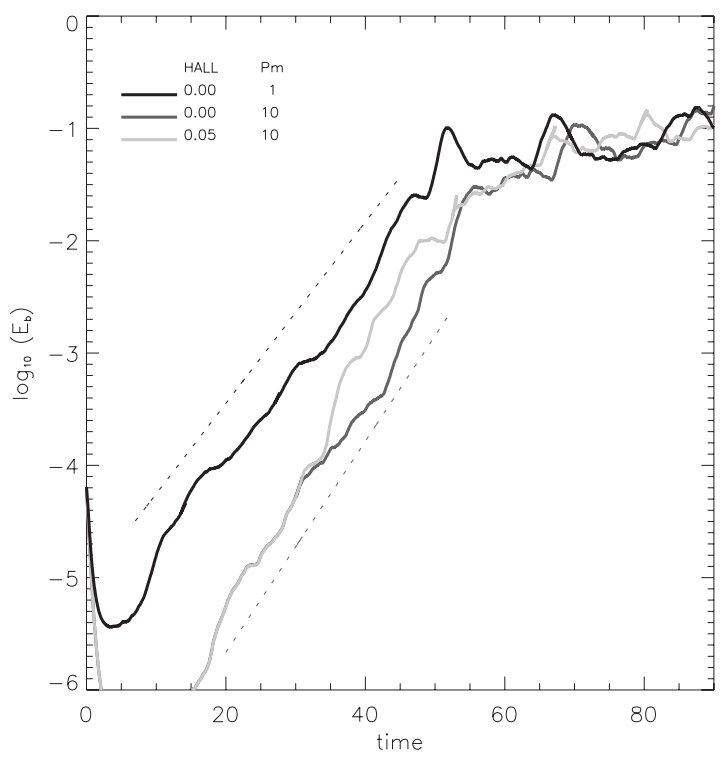

FIG. 10. Magnetic energy vs time for the three runs as labeled.

In Fig. 9 we show the energy spectra for three different runs (labeled). In the top frame we repeat the spectra from the purely MHD run with $\mathrm{Pm}=1$ as a reference. In the central frame we show the spectra for an MHD run (i.e., $\epsilon=0$ ), but with $\mathrm{Pm}=10$. The thick trace corresponds to the totalenergy spectrum at $t=72$, corresponding to the saturation of the dynamo. The various thin curves correspond to the magnetic energy spectrum at the successive times $t=18,36$, and 72 from bottom to top. In the large-Pm regime, the magnetic field grows in the subviscous region of the spectrum. It is apparent that for $\mathrm{Pm}=10$ most of the energy at small scales (i.e., large $k$ 's) is magnetic. These results are consistent with those reported in Ref. [11] from very similar nonhelical simulations. The Kazantsev spectrum $E_{k} \approx k^{3 / 2}$ is also a good approximation at large scales, although at large Pm it is less surprising since the kinetic energy is more confined toward the small- $k$ spectral region.

The lower panel in Fig. 9 corresponds to a simulation with moderate Hall value $(\epsilon=0.05)$ and large magnetic Prandtl number $(\mathrm{Pm}=10)$. By comparison with the case displayed in the central panel $(\epsilon=0$ and $\mathrm{Pm}=10)$, the dynamo efficiency is larger in the presence of the Hall effect, as also confirmed in Fig. 10.

Figure 10 shows the growth of magnetic energy in the three simulations in linear-logarithmic scale. In the MHD

TABLE II. Global results for runs with different values of the magnetic Prandtl number Pm. $\epsilon$ is the amplitude of the Hall effect, $E_{b} / E$ is the ratio of magnetic to total energy, $k_{J}$ is the magnetic Taylor wave number for the current density distribution, $D / D_{0}$ is the normalized dissipation rate, and $D_{b} / D$ is the ratio of magnetic to total dissipation.

\begin{tabular}{crrrrrrr}
\hline \hline$\epsilon$ & $\mathrm{Pm}$ & $E$ & $E_{b} / E$ & $k_{J}$ & $D$ & $D / D_{0}$ & $D_{b} / D$ \\
\hline 0.00 & 1 & 0.37 & 0.14 & 23.1 & 0.13 & 0.88 & 0.48 \\
0.00 & 10 & 0.29 & 0.25 & 16.4 & 0.14 & 2.19 & 0.37 \\
0.05 & 10 & 0.26 & 0.19 & 14.3 & 0.11 & 1.79 & 0.21 \\
\hline \hline
\end{tabular}

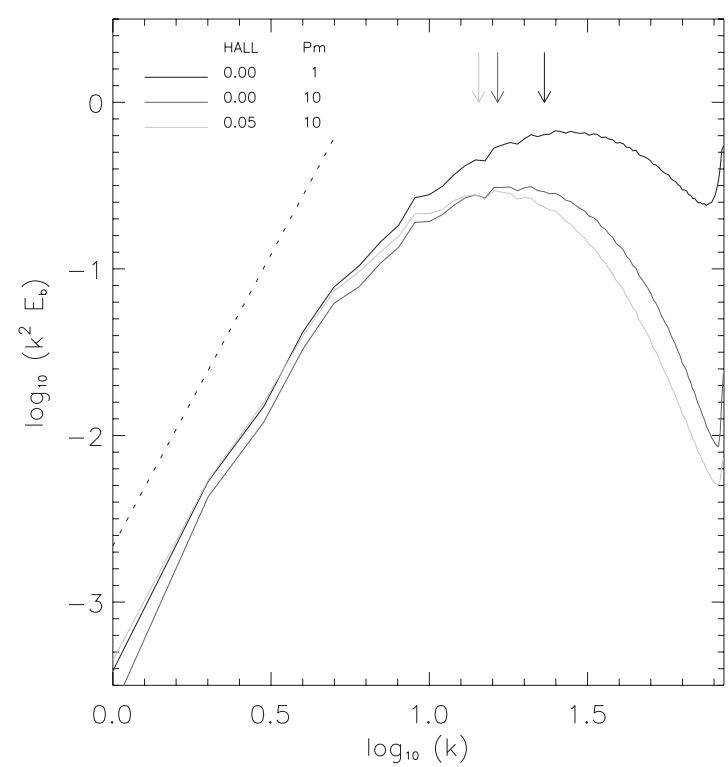

FIG. 11. Spectral distribution of current density, i.e., $k^{2} E_{b}(k)$ vs $k$ for three runs with different values of $\epsilon$ and Pm (labeled). The dotted trace corresponds to the Kazantsev slope $k^{7 / 2}$. The arrows indicate the average wave number $k_{J}$ [see Eq. (6)] for each of the current density distributions.

case, the linear growth rate at large Pm (for the same magnetic diffusivity $\eta$ ) is somewhat larger than in the $\mathrm{Pm}=1$ case, which can be expected as the flow is smoother in the former case and turbulent magnetic diffusion is therefore less effective. In the Hall-MHD case, the linear regime is again found to be followed by a nonlinear stage when the Hall effect becomes relevant and the magnetic field is advected by the electron velocity, as found in the simulations with $\mathrm{Pm}=1$.

Other features of the Hall-MHD small-scale dynamos reported in the $\mathrm{Pm}=1$ simulations can also be identified in the $\mathrm{Pm}=10$ case. As examples, Table II shows saturation values of the total energy, total dissipation, and the ratios of magnetic to total energy and magnetic to total dissipation for the runs in Figs. 9 and 10. In the MHD case, the increase in the magnetic Prandtl number moves the peak of the current density spectrum toward smaller wave numbers (see the values of $k_{J}$ in Table II and Fig. 11). As discussed in Sec. IV, the Hall effect moves this peak further to larger scales.

\section{CONCLUSIONS}

We present results from three-dimensional simulations of small-scale dynamo action for magnetic Prandtl numbers $\mathrm{Pm}=1$ and 10 in conducting flows with the Hall effect. This effect is believed to be non-negligible in sufficiently diffuse media, and its relevance has been recognized in various astrophysical, space, and laboratory plasmas. As a first step toward a better description of dynamo action in such media, only the incompressible Hall-MHD equations were solved, and the inclusion of compressible effects as well as other kinetic effects such as ambipolar diffusion is left for future studies. 
However, the inclusion of only the Hall effect acting at the smallest relevant dynamical scales of the flow gives rise to measurable differences with previous studies of dynamo action. A magnetic nonlinear regime is identified when the magnetic field (and the current density) becomes large enough to differentiate the electron velocity from the bulk flow velocity. After saturation, differences in the stationary level of magnetic energy and in the total and magnetic energy dissipation rates are obtained (as well as the normalized energy dissipation rates $\left.D / D_{0}\right)$, depending on the amplitude of the Hall effect. Finally, the peak of the current density spectrum is found to be dependent on the strength of the Hall term, with its peak moving toward larger scales (smaller wave numbers) as the Hall scale is increased.

By studying the detailed transfer of energy among fields and scales, we observe that the effect of the Hall term is twofold: it transfers energy toward larger scales for scales larger than the Hall length, and it transfers energy toward smaller scales for scales smaller than this length. The modification of the energy flux resulting from this transfer is consistent with the observed changes in the saturation values of energy and dissipation rate observed in our simulations.

\section{ACKNOWLEDGMENTS}

The authors acknowledge support from UBACYT Grants No. X468/08, No. X469/08, and No. X092/08, and from PICT Grants No. 2005-33370, No. 2007-02211, and No. 2007-00856. The National Center for Atmospheric Research is sponsored by the National Science Foundation.
[1] S. R. Spangler, Space Sci. Rev. 99, 261 (2001).

[2] A. H. Minter and S. R. Spangler, Astrophys. J. 458, 194 (1996).

[3] P. Schuecker et al., Astron. Astrophys. 426, 387 (2004).

[4] A. Pouquet, U. Frisch, and J. Leorat, J. Fluid Mech. 77, 321 (1976).

[5] M. Meneguzzi, U. Frisch, and A. Pouquet, Phys. Rev. Lett. 47, 1060 (1981).

[6] A. Brandenburg, Astrophys. J. 550, 824 (2001).

[7] D. O. Gómez and P. Mininni, J. Atmos. Sol.-Terr. Phys. 67, 1865 (2005).

[8] A. P. Kazantsev, Sov. Phys. JETP 26, 1031 (1968).

[9] A. A. Schekochihin, S. C. Cowley, J. L. Maron, and J. C. McWilliams, Phys. Rev. Lett. 92, 054502 (2004).

[10] A. Schekochihin, S. C. Cowley, J. L. Maron, and L. Malyshkin, Phys. Rev. E 65, 016305 (2001).

[11] Nils Erland L. Haugen and A. Brandenburg, Phys. Rev. E 70, 036408 (2004).

[12] P. D. Mininni, Y. Ponty, D. C. Montgomery, J.-F. Pinton, H. Politano, and A. Pouquet, Astrophys. J. 626, 853 (2005).

[13] A. Schekochihin, S. C. Cowley, S. F. Taylor, J. L. Maron, and J. C. McWilliams, Astrophys. J. 612, 276 (2004).

[14] A. Schekochihin, J. L. Maron, S. C. Cowley, and J. C. McWilliams, Astrophys. J. 576, 806 (2002).

[15] Y. Ponty, P. D. Mininni, D. C. Montgomery, J.-F. Pinton, H. Politano, and A. Pouquet, Phys. Rev. Lett. 94, 164502 (2005).

[16] A. B. Iskakov, A. A. Schekochihin, S. C. Cowley, J. C. McWilliams, and M. R. E. Proctor, Phys. Rev. Lett. 98, 208501 (2007).

[17] R. Monchaux et al., Phys. Rev. Lett. 98, 044502 (2007).

[18] T. Sano and J. M. Stone, Astrophys. J. 570, 314 (2002).

[19] E. G. Zweibel, Astrophys. J. 567, 962 (2002).

[20] A. Brandenburg and K. Subramanian, Astron. Astrophys. 361, L33 (2000).

[21] S. A. Balbus and C. Terquem, Astrophys. J. 552, 235 (2001).

[22] P. D. Mininni, D. O. Gómez, and S. M. Mahajan, Astrophys. J. 567, L81 (2002).

[23] X. H. Deng and H. Matsumoto, Nature (London) 410, 557 (2001).
[24] M. Øieroset, T. D. Phan, M. Fujimoto, R. P. Lin, and R. P. Lepping, Nature (London) 412, 414 (2001).

[25] F. S. Mozer, S. D. Bale, and T. D. Phan, Phys. Rev. Lett. 89, 015002 (2002)

[26] V. V. Mirnov, C. C. Hegna, and S. C. Prager, Plasma Phys. Rep. 29, 566 (2003).

[27] M. Yamada, H. Ji, S. Hsu, T. Carter, R. Kulsrud, Y. Ono, and F. Perkins, Phys. Plasmas 4, 1936 (1997)

[28] Y. Ren, M. Yamada, H. Ji, S. Dorfman, S. P. Gerhardt, and R. Kulsrud, Phys. Fluids 15, 082113 (2008).

[29] P. D. Mininni, D. O. Gómez, and S. M. Mahajan, Astrophys. J. 584, 1120 (2003).

[30] P. D. Mininni, D. O. Gómez, and S. M. Mahajan, Astrophys. J. 587, 472 (2003).

[31] N. Kleeorin and I. Rogachevskii, Phys. Rev. E 50, 493 (1994).

[32] T. Passot, E. Vazquez-Semadeni, and A. Pouquet, Astrophys. J. 455, 536 (1995).

[33] P. D. Mininni, D. O. Gómez, and S. M. Mahajan, Astrophys. J. 619, 1019 (2005).

[34] A. Brandenburg and K. Subramanian, Phys. Rep. 417, 1 (2005).

[35] S. Servidio, W. H. Matthaeus, M. A. Shay, P. A. Cassak, and P. Dmitruk, Phys. Rev. Lett. 102, 115003 (2009).

[36] P. Dmitruk and W. H. Matthaeus, Phys. Plasmas 13, 042307 (2006).

[37] Y. Kaneda and T. Ishihara, J. Turbul. 7, 20 (2006).

[38] P. D. Mininni and A. Pouquet, Phys. Rev. E 80, 025401 (2009).

[39] L. Turner, IEEE Trans. Plasma Sci. 14, 849 (1986).

[40] M. Verma, Phys. Rep. 401, 229 (2004).

[41] A. Alexakis, P. D. Mininni, and A. Pouquet, Phys. Rev. E 72, 046301 (2005).

[42] P. D. Mininni, A. Alexakis, and A. Pouquet, J. Plasma Phys. 73, 377 (2007).

[43] S. M. Mahajan and Z. Yoshida, Phys. Rev. Lett. 81, 4863 (1998).

[44] J. Birn et al., J. Geophys. Res. 106, 3715 (2001).

[45] D. Smith, S. Ghosh, P. Dmitruk, and W. H. Matthaeus, Geophys. Res. Lett. 31, L02805 (2004). 